\title{
Epidemiological Survey of Candida Species Isolated from Immunocompromised Hosts at the University of Nigeria Teaching Hospital, Enugu State
}

Uchegbu UN ${ }^{1 *}$, Onyemelukwe $\mathrm{NF}^{2}$, Amah $\mathrm{HC}^{3}$, Uche-Uchegbu N ${ }^{4}$, Uduji HI ${ }^{3}$, Ndukwe $\mathrm{CK}^{5}$ and Oparaocha $\mathrm{SC}^{6}$

${ }^{1}$ Department of Medical Laboratory Services, Federal Medical Centre, Nigeria

${ }^{2}$ Department of Medical Laboratory Science, College of Medicine, University of Nigeria Enugu

Campus, Nigeria

${ }^{3}$ Department of Medical Laboratory Science, Imo State University, Nigeria

${ }^{4}$ Department of Internal Medicine, Federal Medical Centre, Nigeria

${ }^{5}$ Research Assistant, National Orthopaedic Hospital Enugu, Nigeria

${ }^{6}$ Research Assistant, Nnamdi Azikiwe University Teaching Hospital, Nigeria

\section{Research Article \\ Volume 3 Issue 5}

Received Date: October 01, 2020

Published Date: October 15, 2020

DOI: $10.23880 /$ jqhe-16000182

*Corresponding author: Uchegbu UN, Department of Medical Laboratory Services, Federal Medical Centre, Owerri, Imo State, Nigeria, Tel: +2348037090618; Email: druchegbujnr@yahoo.com

\section{Abstract}

Candida species are excessively abundant in nature as free living organisms with the ability to infect humans and animals worldwide. They are notorious for their unbending resistance to the common azoles used in the treatment of Candidiasis in immunocompromised patients, hence making correct identification indispensable. In this study, 315 clinical specimens were collected from patients with one form of immunosuppression or the other while 150 swab samples were collected from the patients' immediate environment. The samples were cultured on SDA and ChromAgar for identification of Candida species. The isolates were further identified to the species level using conventional methods based on their Gram reactions and Germ tube test. 158 biological specimens yielded Candida species; which represents an incidence rate of (50.2\%), while $34(22.7 \%)$ of the environmental samples yielded Candidal growth. The identified isolates are; Candida metapsilosis, C. albicans, C. parapsilosis, C. orthopsilosis and C. tropicalis. These findings about the environmental pathogens are essential as they will help in developing effective preventive and therapeutic strategies against invasive candidiasis from the $C$. albicans and nonalbicans Candida (NAC) pathogens. More so, the identification of the Candida species from the environment gives an idea of the development of possible re-infection of the immunocompromised patients by the organisms in the environment. The environmental candidiasis may also account for the much talked about resistance to the common antifungal drugs.

Keywords: Candida Species; Patients' Beds; Hospitals; Humans

\section{Introduction}

Candida species are excessively abundant in nature as free living organisms with a peculiar arrangement of nuclear materials, presence or absence of mitochondria and endoplasmic reticulum. Candida is a genus of yeast and a common cause of fungal infections worldwide [1]. Candida species are earlier confined to the human and 
animal reservoirs but they are now frequently recovered from the hospital environs such as from operating couches (Tables), patients' beds, wall surfaces of wards, theatres (a square area marked out on the four corners of the walls), door handles of theatres, ICUs, pocket areas of the theatre gowns, Nurses' uniforms and ward coats $(2 \mathrm{~cm}$ from the mouth/lid of the pocket upwards), ultra sound scan (USS) probes, laboratory benches, air samples, foods, table tops, air-conditioners, floors, walls, respirators and other medical devices and personnel [2]. These species are notorious for their resistance to the common azoles which are used in the treatment of candidiasis. This makes it pertinent to carry out a research into the isolation of Candida from the environment and humans, especially the immunocompromised patients [3].

The infection caused by Candida species is called candidiasis, though some species are harmless commensals of humans hosts [4]. However, when mucosal linings are disrupted or the immune system compromised, they can invade and cause diseases [5]. The steady increase in the problems of mucosal and systemic candidiasis reflect the enormous rise in the number of patients at risk and the increased opportunity that exists for Candida species to invade tissues that are normally resistant to invasion. Candida species are true opportunistic pathogens that exploit some normal physiological phenomena and recent technological advances to gain access to the circulation and deep tissues [6].

The incidence of Candida infections have increased substantially in recent times. Therefore, early detection of candidiasis and the correct identification of the Candida species are pre-requisites for the appropriate therapeutic intervention [7]. Candida pathogenicity is facilitated by a number of virulence factors, the most important of which are those for adherence to host tissues and medical devices, biofilm formation and secretion of hydrolytic enzymes called, proteases, phospholipases and haemolysins $[8,9]$.

The primary factor in the fungal colonization of human tissues is adherence to host surface; this process is controlled and induced by several cell-mediated activities induced by the fungus and environment [10]. Biofilms cause clinical problems because they increase resistance to antifungal therapy. Extracellular hydrolytic enzymes play an important role in adherence, tissue penetration, invasion and the destruction of host tissues [11]. Unfortunately, diagnosis of invasive candidiasis is still problematic because clinical symptoms are non-specific and conventional assays are not satisfactorily precise and may take several days to obtain results. Blood cultures which are assumed to be the most reliable marker of invasive candidiasis are commonly negative, whereas positive cultures form other sites may represent colonization [12]. Also, blood culture requires 2-5 days for correct identification.

The most often identified species has always been Candida albicans. However, the incidence of non-albicans Candida species has been increasing with the changing spectrum of patients and these species have also been associated with higher mortality. C. parapsilosis persists in hospital environments, thereby enhancing the chances of acquiring nosocomial infections. It colonizes the skin of patients and it is able to form biofilms on plastics resulting in candidaemia in patients with in-dwelling venous catheters [13].

Epidemiological investigations of outbreaks in hospitals are required to identify the source of infection, route of transmission and how to eradicate it. Such investigations require accurate strain typing because in candidaemia it is problematic to distinguish between endogenous and exogenous sources of infection from another infected patient(s), or even healthcare workers, as the case may be [14]. In healthy and immunocompetent individuals, Candida does not usually give rise to diseases as its growth is suppressed by other micro biota and the host defense mechanisms. However, in a situation where there is alteration in the mucosal barriers or the immune system is compromised, they can invade and cause disease [15].

Candida species are considered important pathogens due to their versatility and ability to survive in various anatomical sites [16]. In the early 1980s, fungi emerged as major causes of nosocomial infections, mainly affecting immunocompromised patients or those who were hospitalized for long periods due to serious underlying diseases. Prolonged ICU stay and hospitalization are also important. Akpan, et al. [17] evaluated the impact of invasive fungal infection in surgical patients with severe Candidal infections are a major problem, especially among the immunosuppressed people. Oral infections with yeasts are more frequent in diabetic patients, but some results remain controversial and contradictory. A great number of reports suggest that Candida albicans is the commonest species that inhabit the oral mucosa of diabetic patients and the highest rate of colonization occurs in diabetic patients with poor glycaemic control [16]. Oral candidal infection has been associated with cigarette smoking and use of dentures in adults with diabetes mellitus and reduction in salivary flow in these patients increases the incidence of oral candidiasis. The epidemiology of oral Candidal infection in diabetic patients seems to be complex, resulting from a combination of many predisposition factors [17].

Due to high production of progesterone during pregnancy, the normal functioning of neutrophils is impaired 
which in turn increases Candida colonization and infection. In addition, high production of oestrogen during pregnancy has its own impact on immunoglobulin secretion in the vagina which results in mucosal surface defense failure [5]. Generally, the main reason for increased vaginal candidiasis in pregnant women is due to elevation of reproductive hormones during pregnancy. High levels of reproductive hormones during pregnancy provide an increased amount of glycogen in the vagina which enhances growth of Candida species because glycogen serves as a carbon source for Candida growth. In addition, oestrogen enhances adherence of yeast to the vagina by reducing immunoglobulin secretion and fungal inhibition activity of the epithelial cells [18]. The clinical manifestation of the disease is characterized by vaginal pruritis, thick curd/cheese-like vaginal discharge, itching, redness, burning, swelling and pain during walking and urination [19]. These clinical signs and symptoms, though not specific, are important for the diagnosis of vaginal candidiasis. Pregnant women with such clinical manifestations should be treated as early as possible because candidiasis is reported as a risk factor for pre-term birth. In complicated candidiasis, non-albicans Candida species are common and the disease is commonly seen in diabetic, debilitated or immunocompromised women. The outcome of complicated vaginal candidiasis is psychosexual problems (e.g. feeling unclean or not wanting to have sex) and depression [20].

A neonate is a baby who is 4 weeks old or younger. A neonate is also called a new born. The neonatal period is the first 4 weeks of a child's life. It is a time when changes are very rapid. Systemic fungal infections, previously considered to be a rare complication, occur in as many as $5 \%$ low birth weight babies. They are even more frequently diagnosed in very low-birth weight babies (VLBW) receiving intensive care. About $20 \%$ of babies weighing less than $1000 \mathrm{~g}$ develop invasive fungal infections. The overall mortality rate for disseminated fungal infections in this group of babies is very high, often approaching 50\% [21].

Evidences abound to show that Candida species can be acquired from both clinical and environmental sources. The understanding of the epidemiology of candidiasis has grown appreciably over the past years. Rapid changes in the rates of infection, potential risk factors and emerging new species in the aetiologic frequencies demand continued and close surveillance in our hospitals and environs. Recent studies, performed years ago have broadened the epidemiologic understanding of nosocomial candidiasis from a single endogenous infection to one that may be exogenously acquired through the hands of health care givers, biomaterials and inanimate environment including the walls of theatres, wards and the wears of health care givers. While these information are documented and very well at hand in developed countries, the knowledge in developing countries including Nigeria is either conflicting, biased, inadequate or completely inexistent. It is therefore pertinent that studies are undertaken to define more accurately the emergence of new species, specificity of old ones, reservoirs, mode of transmission, relationship with immunocompromised patients, the best method of identifying these Candida species and their exact extent of involvement in nosocomial infection in this country and South East Nigeria, in particular.

Therefore, there is need to carry out this research to unravel the extent of damage these Candida species have caused in our environment due to misdiagnosis and the unavoidable contact of the organism (Candida) with the patients or the healthcare giver or even medical devices that in turn transmit these organisms to the patients in form of re-infection in the course of healthcare delivery. Therefore, this study is aimed at determining the prevalence and distribution pattern of Candida species from clinical and environmental sources of each of the Healthcare facilities and to assess the risk factors involved.

\section{Materials and Methods}

\section{Study Area}

The samples were collected from the University of Nigeria Teaching Hospital (UNTH) Enugu. The samples collected were processed in the Microbiology Laboratory of the hospital. The University of Nigeria Teaching Hospital is a tertiary healthcare facility that provides healthcare services to the individuals within and outside its immediate community. The inhabitants of the state are mainly farmers, traders and/or public servants with a population of 3 million [21].

\section{Study Design}

It is a prospective cross-sectional study which was undertaken within the healthcare facilities. The clinical specimens were collected from patients with one form of immunosuppression or the other. The conditions considered included; HIV/AIDS, Diabetes mellitus, Pregnancy and Neonates/infants (0-24weeks).

The study considered two (2) pathological conditions and two (2) normal physiological conditions that reduce immunity of the individuals.

\section{Sample Collection}

- Clinical samples: HIV/AIDS-sputum, diabetics-early morning urine sample, pregnancy-High Vaginal Swab (HVS) and neonates-stool samples. 
The stool sample was earlier cultured onto SalmonellaShigella Agar (SSA), MacConkey agar (MAC) and Deoxycholate Citrate Agar (DCA). And was found to contain no bacteria, hence the screening for Candida species.

- Environmental samples: These included the swabs of the surface areas of operating couches (tables), patients' beds, wall surfaces of wards, ICUs and theatres (a square area marked out on the four corners of the walls), door handles of theatres, pocket areas of the theatre gowns, Nurses' uniforms and ward coats $(2 \mathrm{~cm}$ from the mouth/ lid of the pocket upwards), Ultra Sound Scan (USS) probes, laboratory benches and air samples.

- Selection Criteria: HIV patients presenting for the study shall be those attending the clinics or admitted in the wards or ICU; whose CD4 counts are less than or equal to 200 cells $/ \mathrm{ml}$ of blood ( $\leq 200$ cells $/ \mathrm{ml}$ of blood). Pregnant women who are $>6$ weeks gone. Neonates; those who were $0-24$ weeks presenting with oral candidiasis from birth or acquired due to poor immune responses. And, the environmental specimens must include the swabs of all the materials mentioned above that are used in the hospitals for patients care.

\section{Preparation of Culture Media}

Two (2) different media were used for the isolation and identification of Candida species. They are;

1. Sabouraud Dextrose Agar (SDA Biotech): Used for the isolation of the Candida species

2. ChromAgar (TM Biotech): used for the speciation of the yeast cells according to colour pigmentation.

The SDA was measured out according to the manufacturer's instruction and each dissolved in 1Litre $(1000 \mathrm{ml})$ of water.

The preparation was heated for 10 minutes and $0.05 \mathrm{~g}$ of Chloramphenicol dissolved in $10 \mathrm{ml}$ of ethanol was added into both. Each mixture was autoclaved at $121^{\circ} \mathrm{C}$ for 15 minutes. About 15-20 ml of each preparation was dispensed into different Petri-dishes and allowed to solidify before use.

The ChromAgar medium is called chromogenic Candida Agar. Unlike other media discussed above, this Agar (medium) contains among other ingredients; a chromogenic mixture, which is a colour differential ingredient that defines specific colours for particular species of Candida. It already has 0.05 $\mathrm{g} / \mathrm{L}$ of Chloramphenicol supplemented in the agar base.

$42.72 \mathrm{~g}$ of the agar was mixed in 1Litre $(1000 \mathrm{~mL})$ of water and the mixture gently heated over a Bunsen flame to hasten complete dissolution of the powder in water. The preparation does not need autoclaving for sterilization. The preparation was allowed to cool to $50^{\circ} \mathrm{C}$ and dispensed into Petri-dishes. After the medium solidified, it was ready for use.

\section{Processing of Samples}

(A.) A total of 315 clinical specimens made up of 108 HVS from pregnant women, 12 stool samples from neonates, 127 sputum samples from HIV/AIDS patients and 68 urine (biological) specimens from diabetics, respectively, were used for the study.

(B.) In the same vein, 150 hospital environmental swab samples were collected from the wards, theatres, ICUs, Laboratories and other machines / equipments and wears used in patients' treatment in the same facility.

Each of the samples mentioned in A and B above was cultured (seeded) unto the SDA and the Chrom Agar; and incubated at $37^{\circ} \mathrm{C}$ for 72 hours with daily check for possible growth. The air samples were collected by exposing the media to the air of the collection areas for 24 hours, after which they were incubated alongside other samples and daily observed for obvious growth. While the Chrom Agar helped to differentiate the Candida species by color pigmentation, the SDA gave luxuriant growths of the Candida species with some forming chlamydospores.

\section{Microscopy: Wet preparation (KOH mount)}

Each of the samples from A and B above (after culture) was emulsified in 3-5 drops of $10 \% \mathrm{KOH}$ on a grease free slide. The preparation was covered with cover slip and viewed under the microscope with $\mathrm{x} 10$ and $\mathrm{x} 40$ objectives for yeasts cells and/or chlamydospores.

\section{Identification And Characterization \\ - Gram reactions, and \\ - Germ tube tests}

\section{Gram Reactions}

The Candida colonies were emulsified in a drop of water on a grease-free slide and allowed to air dry. The preparation was flooded with crystal violet and allowed for 60 seconds. The stain was tipped off and the slide was flooded with Lugol's iodine and allowed for 30 seconds and then rinsed in water. The slide was further flooded with acetone for a few seconds and then tipped off. The slide was counter stained with safranin for 30 seconds and later rinsed off in water. It was allowed to air dry and viewed under x100 (oil immersion) objective lens. 


\section{Germ Tube Test}

A loopful of colony of the Candida isolates was emulsified into 2-3 drops of human serum. The mixture was incubated at $37^{\circ} \mathrm{C}$ for $2-4$ hours with hourly checks. A sprouting hyphae under $\mathrm{x} 40$ objective lens of the microscope indicated positive colonies for Candia albicans, C. dubliniensis, C. stellatoides and others that are positive for Germ tube test.

\section{Results}

315 clinical specimens (sputum, urine, HVS and stool samples) from 4 immunocompromised conditions (HIV/ AIDS, Diabetes mellitus, Pregnancy and Neonates) and 150 swab samples from the hospital environments which included the theatres, wards, ICUs, Laboratories, air samples and other health care delivery materials from the hospital in the South Eastern Nigeria were screened for the presence of Candida species. This is to ascertain to what extent the patient environment helps in the infection and re-infection of the patients especially the immunocompromised.

\section{Discussion}

Scholars have over time studied and opined that the infective process initiated by Candida species has been upgraded by a number of notable virulence factors that confers on them certain immuno-invasive potentials in order to thrive in their host(s) and perpetrate health challenges. Such factors include adhesins for attachment to the host's tissues and medical equipment, the formation and settling of biofilms on medical devices found within the patient's environment and the ability to secrete hydrolytic enzymes that attack and break down the host's defense mechanisms in a bid to initiate an infection. This concern underscores the aim of this study; trying to isolate and identify Candidal isolates from both the immunocompromised patients and their intimate environment. However, the pressing need to rightly identify the isolates has not been without limitations that common with the conventional methods of identification, thereby compounding the problems of misdiagnosis and the unchecked re-infection.

Table 1 shows the results obtained from culturing the specimens collected from the immunocompromised patients in University of Nigeria Teaching Hospital (UNTH), Enugu South Eastern Nigeria. Out of the 315 specimens collected from these human sources, 158(50.2\%) yielded fungal growth, while $157(49.8 \%)$ yielded no obvious growth of any Candida species. The identification of the aforementioned isolates was from conventional methods which corroborates with the work of Pfaller, et al. [13] where he recorded that immunosuppression is a favorable condition for fungal colonization in immunocompromised patients. Additionally, the study confirms that immunosuppression supports the isolation of fungal species from most of the specimens as seen in UNTH Enugu, where 66 (51.9\%) out of the 127 HIV patients used for the study, yielded Candida isolates. Pappas, et al. [16] said something to support this notion. He was of the opinion that Candida species are opportunistic, hence maximize every and any opportunity to gain access into deep tissues when the immune responses are jeopardized.

\begin{tabular}{|c|c|c|c|c|c|c|c|c|c|}
\hline $\begin{array}{l}\text { Health Care } \\
\text { Facility }\end{array}$ & $\begin{array}{l}\text { HIV/AIDS } \\
\text { (Sputum) }\end{array}$ & (\%) & $\begin{array}{c}\mathrm{D} / \mathrm{M} \\
\text { (Urine) }\end{array}$ & (\%) & $\begin{array}{l}\text { Pregnancy } \\
\text { (HVS) }\end{array}$ & (\%) & $\begin{array}{l}\text { Neonates } \\
\text { (Stool) }\end{array}$ & (\%) & $\begin{array}{l}\text { Total Candida } \\
\text { isolates }\end{array}$ \\
\hline UNTH, Enugu & $127(66)$ & 52 & $68(32)$ & 47.1 & $108(56)$ & 51.9 & $12(4)$ & 33 & $158(50.2 \%)$ \\
\hline Total & $127(40.3)$ & & $68(21.6)$ & & $108(34.3)$ & & $12(3.81)$ & & $315(100 \%)$ \\
\hline
\end{tabular}

Table 1: Clinical specimens and incidence of the Candidal load from immunocompromised conditions in UNTH, Enugu State, Nigeria.

In Table 2, the Candida species were isolated using conventional methods from the environmental sources; the medical devices and other environmental surfaces around the patient within the healthcare facility. Out of the 150 samples collected from the wards, theatres, ICUs, wears, laboratories and other medical devices at UNTH Enugu, 34 samples yielded Candida species which represents (22.7\%) of the samples size used for this study. From this study, it was recorded that a good percentage of the environmental samples yielded Candida species, except the air samples obtained from the ICU. That means, the air current in these wards where these patients are attended to can become a potential source of infection and can induce the re-infective processes commonly seen among immunocompromised patients. These findings so much agrees with the work of Pfaller, et al. [13]: he reported that additional infections do arise by reasons of exogenous contamination through the healthcare workers, medical devices, biomedical instruments and the very immediate surroundings of these immunocompromised patients. He continued by saying that this can bring about hospital-acquired infections, especially among patients' with any of the debilities considered in this study. 


\section{Journal of Quality in Health care \& Economics}

\begin{tabular}{|c|c|c|c|c|c|c|c|c|c|c|c|c|c|c|c|c|c|c|}
\hline $\begin{array}{c}\text { Health } \\
\text { Care } \\
\text { Facility }\end{array}$ & $\begin{array}{c}\text { No of } \\
\text { samples }\end{array}$ & $\begin{array}{c}\text { Operating } \\
\text { tables }\end{array}$ & $\begin{array}{c}\text { Patients' } \\
\text { beds }\end{array}$ & $\begin{array}{c}\text { Wall } \\
\text { surface } \\
\text { Wards }\end{array}$ & $\begin{array}{c}\text { Wall } \\
\text { surface } \\
\text { ICUs }\end{array}$ & $\begin{array}{c}\text { Wall } \\
\text { surface } \\
\text { Theatres }\end{array}$ & $\begin{array}{c}\text { Door } \\
\text { handle } \\
\text { wards }\end{array}$ & $\begin{array}{c}\text { Door } \\
\text { handle } \\
\text { Theatres }\end{array}$ & $\begin{array}{c}\text { Door } \\
\text { handle } \\
\text { ICUs }\end{array}$ & $\begin{array}{c}\text { Pocket } \\
\text { areas of } \\
\text { Nurses } \\
\text { uniform }\end{array}$ & $\begin{array}{c}\text { Pocket } \\
\text { areas } \\
\text { of ward } \\
\text { coats }\end{array}$ & $\begin{array}{c}\text { USS } \\
\text { probes }\end{array}$ & $\begin{array}{c}\text { Lab } \\
\text { benches }\end{array}$ & $\begin{array}{c}\text { Air } \\
\text { sample } \\
\text { wards }\end{array}$ & $\begin{array}{c}\text { Air } \\
\text { sample } \\
\text { theatres }\end{array}$ & $\begin{array}{c}\text { Air } \\
\text { sample } \\
\text { ICUs }\end{array}$ & $\begin{array}{c}\text { Total } \\
\text { Candida } \\
\text { isolates }\end{array}$ & (\%) \\
\hline $\begin{array}{l}\text { UNTH, } \\
\text { Enugu }\end{array}$ & 150 & $10(3)$ & $10(2)$ & $10(2)$ & $10(1)$ & $10(1)$ & $10(2)$ & $10(1)$ & $10(3)$ & $10(3)$ & $10(2)$ & $10(4)$ & $10(3)$ & $10(4)$ & $10(2)$ & $10(1)$ & 34 & $22.70 \%$ \\
\hline Total & 150 & $3(8.8)$ & $2(5.8)$ & $2(5.8)$ & $1(2.9)$ & $1(2.9)$ & $2(5.8)$ & $1(2.9)$ & $3(8.8)$ & $3(8.8)$ & $2(5.8)$ & $4(11.8)$ & $3(8.8)$ & $4(11.8)$ & $2(5.8)$ & $1(2.9)$ & 34 & $100 \%$ \\
\hline
\end{tabular}

Table 2: Sources of Environmental Candida from UNTH, Enugu.

However, the methodology adopted in this study slightly veered off course from the normal in identifying the species of Candida species isolated using Chrom Agar. Owing to the fact that way before now, most of the authors who worked on related studies identified $C$. albicans as the common culprit associated with candidiasis. This study identified $C$. tropicalis as the most offending Candida species followed by C. albicans and $C$. metapsilosis from the studied samples.

Nevertheless, this study focused on the infective significance of the Candida species found within the immediate surroundings of these immune-debilitated patients which can possibly cause infection or initiate reinfections due to continuous contact with the patient's external environment via the healthcare givers or from medical devices. Either ways, thoughts about the infective significance of viable biofilms that can initiate an infective process in an immunocompromised patient was considered than the high percentage of isolates that exist as fomites or as aerosols that cannot cause any infections even in the immunocompromised patients understudied herein.

Evidences abound putting forth that the non-albicans candidiasis has gotten enough points as aetiologic agents of infections and re-infection. Also, a better understanding of potential reservoirs of these Candida species and their modes of transmission to the immunocompromised patients had been established before now. These findings are essential as they will help in developing effective preventive and therapeutic strategies against invasive candidiasis from the C. albicans and non-albicans Candida (NAC) pathogens.

In all, 5 different fungal organisms were isolated from both clinical specimens with different percentage occurrence. From analysis, Candida metapsilosis occurred 1(12.5\%), C. albicans $1(12.5 \%)$, C. parapsilosis $2(25 \%)$, C. orthopsilosis $2(25 \%)$, and C. tropicalis 2(25\%).

From Table 3 above, it is observed that Candida tropicalis has the highest frequency in both clinical specimens. Since these samples are from the immediate environment of the patient, cross infection may be possible in the patients who have compromised immunity hence, the preponderance of nosocomial infection due to Candida species among hospitalized patients.

\begin{tabular}{|c|c|c|c|c|}
\hline Number & Isolate & Source & Number of isolates & Percentage (100\%) \\
\hline 1 & C. metapsilosis & Clinical & 1 & $12.50 \%$ \\
\hline 2 & C. albicans & Clinical & 1 & $12.50 \%$ \\
\hline 3 & C. parapsilosis & Clinical & 2 & $25 \%$ \\
\hline 4 & C. orthopsilosis & Clinical & 2 & $25 \%$ \\
\hline 5 & C. tropicalis & Clinical & 2 & $25 \%$ \\
\hline Total & & & $\mathbf{8}$ & $\mathbf{1 0 0 \%}$ \\
\hline
\end{tabular}

Table 3: Shows the Number of Yeast species isolated from the Clinical Specimens.

The findings of this study is corroborated by the 2015 study of Colombo, et al. [20] which stated that $C$. tropicalis is one of the most common colonizers of the human skin, female genitourinary tract and can be transmitted between healthcare workers and patients especially in environments such as hospitals [19]. It is the second most virulent Candida species and can significantly affect by spreading through the weakened immune system host.

\section{References}

1. Manolaki D, Velmahos G, Kourkompetas T, Chang Y, Alam HB, et al. (2010) Candida infection and colonization among trauma patients. Virulence 1(5): 367-375.

2. Ferreira AV, Prado CG, Carvalho RR, Dias KST, Dias ALT (2013) Candida albicans and Non-C. albicans Candida 
species: comparison of biofilm production and metabolic activities in biofilms and putative virulence properties of isolates from hospital environments and infections. Mycopathologia 175(3-4): 265-272.

3. Jha B, Dey S, Tamang MD, Joshy ME, Shivananda PG, et al. (2006) Characterization of Candida species isolated from cases of lower respiratory tract infection. Kathmandu University Medical journal 4(3): 290-294.

4. Adebiyi OE, Oluradun A, Daniel OJ, Oritogun KS, Fasanmade AA (2016) Prevalence of Vulvovaginal Candidiasis among Women with Diabetes Mellitus in Oyo State, Nigeria. Annal of Health Research 24: 54-56.

5. Kourkompetis, Themistoklis K (2011) The effect of cumulative length of hospital astys of antifungal resistance of candida strains isolated from critically ill surgical patients. Mycopathologia171(2): 85-91.

6. Pappas PG, Kauffman CA, Andes D, Benjamin DK, Calendra $\mathrm{TF}$, et al. (2009) Clinical guidelines for the management of candidiasis; update by the Infectious Diseases Society of America. Clinic of Infectious Disease 48(5): 503-535.

7. Tamura NK, Negri MF, Borassoli LA, Svidzinski TI (2007) Virulence Factors for Candida spp recovered from intravascular cathers and hospital workers hands. Rev Soc Bras Med Trop 40(1): 91-93.

8. Silva S, Negri M, Henniques M, Oliviera R, Williams DW, et al. (2011) Candida glabrata, Candida parapsilosis and Candida tropicalis, Biology, Epidemiology Pathogenicity and Antifungal resistance. FEMS Microbial Rev 36(2): 288-305.

9. Chowdhary A, Sharma C, Meis JF (2017) Candida auris: A rapidly emerging cause of hospital-acquired multi-drug resistant fungal infections globally. PloS pathoh 3(5): e1006290.

10. Li YL, Leaw SN, Chen JH, Chang TC (2003) Rapid identification of commonly encountered Candida species directly from blood culture bottles. Journal of clinical Microbiology 41(12): 5660-5664.
11. Verstrepen KJ, Klis FM (2006) Flocullation, adhesion and Biofilm formation in Yeats. Mol microbial 60: 5-15.

12. Kojic EM, Daroniche RO (2004) Candida infections of media devices. Clinical Microbiology Rov 17: 225-267.

13. Pfaller MA, Diekema DJ (2007) Epidemiology of invasive candidiasis: a persistent public health problem. Clinical Microbiology Review 20(1): 133-163.

14. Deepa VK, Vashe KV, Rashmi K, Harish S, Aparna R (2014) Prevalence of vaginal candidiasis in pregnancy among coastal south Indian women. Journal of women health 3(6).

15. Krcmery V, Barnes AJ (2002) Non-albicans candida spp. Causing fungaemia: pathogenicity and antifungal resistance. J Hosp Infect 50: 243-260.

16. Luna MA, Tortoledo EM (1993) Histologic identification and pathologic patterns caused by candida. In candidiasis, pathogenesis, diagnosis and treatment edited by G Bodey Raven Press New York, pp: 21-42.

17. Akpan A, Morgan R (2002) Oral candidiasis. Postgraduate Medical Journal 78: 455-459.

18. Muller FMC, Kasai M, Francesconi A, Brilliante B, Roden M, et al. (1999) Transmission of azole-resistant isogenic strain of Candida albicans among human immunodeficiency-virus-infected family members with oropharyngeal candidiasis. Journal of Clinical Microbiology 37(10): 3405-3406.

19. Moondragon (2006) Obgyn information; gyneacological and obstetrical information discussion.

20. Colombo AL, Guimaraes T, Silva LR (2007) Prospective observational study of candidaemia in Sao Paulo, Brazil: incidence rate, epidemiology and predictors of mortality. Infectious Control Hospital Epidemiology 28(5): 570575.

21. National Population Commission (2006) South Eastern Nigeria. 